\title{
Paste dewatering with the Boozer disc filter
}

\author{
J. Hahn BOKELA GmbH, Germany \\ R. Bott BOKELA GmbH, Germany \\ T. Langeloh BOKELA GmbH, Germany
}

\begin{abstract}
The Boozer disc filter represents the new generation of disc filters, which defines the latest standard in the alumina industry and in the dewatering of coal slurries. Now this new type of disc filter moves into applications of paste dewatering.

In a current modernisation and upgrading project of a gold and copper mine the Boozer disc filter is the chosen technology for dewatering of the gold/copper tailings to make them available for mine backfill. For this application two Boozer L4 disc filters are operated with 4 discs and $176 \mathrm{~m}^{2}$ filter area each. As typical performance rate in this application the Boozer disc filters produce some $500 \mathrm{~kg} / \mathrm{m}^{2} \mathrm{~h}$ of solids throughput with a moisture content of $20 \mathrm{wt} \%$. As a special highlight both Boozer disc filters are equipped with a double dewatering zone which can be activated or deactivated online. Thus, the Boozer provides for reliable operation with constant throughput, constant moisture content and complete discharge of the filter cake even with significant fluctuation of the feed flow properties.
\end{abstract}

The innovative design of the Boozer provides for high throughput and dewatering performance, excellent operational reliability over a broad range of feed conditions, ease of maintenance and high profitability. Compared to filter presses, belt filters or 1970s vintage disc filters the Boozer disc filter is the most economical dewatering technology due to its high capacity, simple design and small footprint.

\section{Introduction}

Filtration of tailings and paste from ore processing is gaining more and more importance. First, the recovery of water back to the process reduces the input of the important resource water. This can already make the difference between "yes we can operate" or "no we can't". Second, the filtered tailings can be dry stacked which is safer, cheaper and environmentally more accepted. And third, especially with mine backfill, the amount of cement to be added can be reduced which may save millions of dollars per year.

In a current modernisation and upgrading project of a gold and copper mine the tailings are made available for mine backfill by filtration. The chosen technology for dewatering of these tailings is the Boozer disc filter which represents the new generation of disc filters.

\section{Processing of the gold/copper tailings after mine upgrade}

Tailings from ore processing are typically thickened to about $50 \mathrm{wt} \%$ prior to filtration. The thickened solids are pumped to disc filters and 10-80 g/t flocculant is added to the filter feed flow. Modern Boozer disc filters are separating the thickened tailing slurry achieving about $500-1,000 \mathrm{~kg} / \mathrm{m}^{2} / \mathrm{h}$ solids throughput and a moisture of about 15-23 wt\% depending on the particle size distribution (PSD) of the feed solids. In the concrete tailing dewatering project of a gold/copper mine the tailing characteristics and target values for filtration are as follows: solids concentration of thickened tailings $>50 \mathrm{wt} \%$, solids throughput is $100 \mathrm{t} / \mathrm{h}$, target moisture of solids after filtration is $23 \mathrm{wt} \%$.

Boozer disc filters can be operated by full automation and allow the operator to react online for changes in the filtration properties of the thickened tailings in order to always maintain the required moisture. This is done through adaptation both of the vacuum in the cake form zone and of the vacuum in the double cake dewatering zone by which means the cake moisture content can be kept on a constant required value. 
Furthermore, modern disc filters are very reliable with regard to cake discharge, no matter how much fines are fed or how the feed solids content changes.

The reliable operation even with high amounts of fines and the constant moisture achieved on modern disc filters allow using the tailings for mine backfill without the need of splitting it into fine or coarse fractions. This reduces the process equipment required and finally the investment cost. Furthermore, it reduces the amount of cement added to the tailings.

\section{Features of the new vacuum disc filter generation}

With the high performance disc filter Boozer BOKELA Company have developed a new generation of big diameter disc filters which have set a new standard for seed filtration in the alumina industry and in the dewatering of coal slurries. Now this new type of disc filter has begun to move into applications of paste dewatering.

The outstanding hydraulic characteristics of the Boozer disc filter were achieved by improving each detail of the filter design leading to extraordinarily high performance capacity, high operational safety and reliability as well as low maintenance and operation costs. The main features of this new disc filter generation can be listed as follows:

- minimised pressure drop leading up to $100 \%$ higher pressure difference at the filter cloth compared to conventional disc filters

- double capacity compared to conventional disc filters

- high filter speed of $6 \mathrm{rpm}$

- high operational reliability and flexibility

- easy maintenance

- fully automatic and safe operation due to superior process philosophy realized in a programmable logic controller system.

Disc diameters range from $1.7 \mathrm{~m}, 4.2 \mathrm{~m}$ to $5.6 \mathrm{~m}$. For filtration of large slurry feed rates such as tailings, a Boozer filter with large disc diameter of $5.6 \mathrm{~m}$ (L-type) is the appropriate filter size which is available with 1 to 4 filter discs.

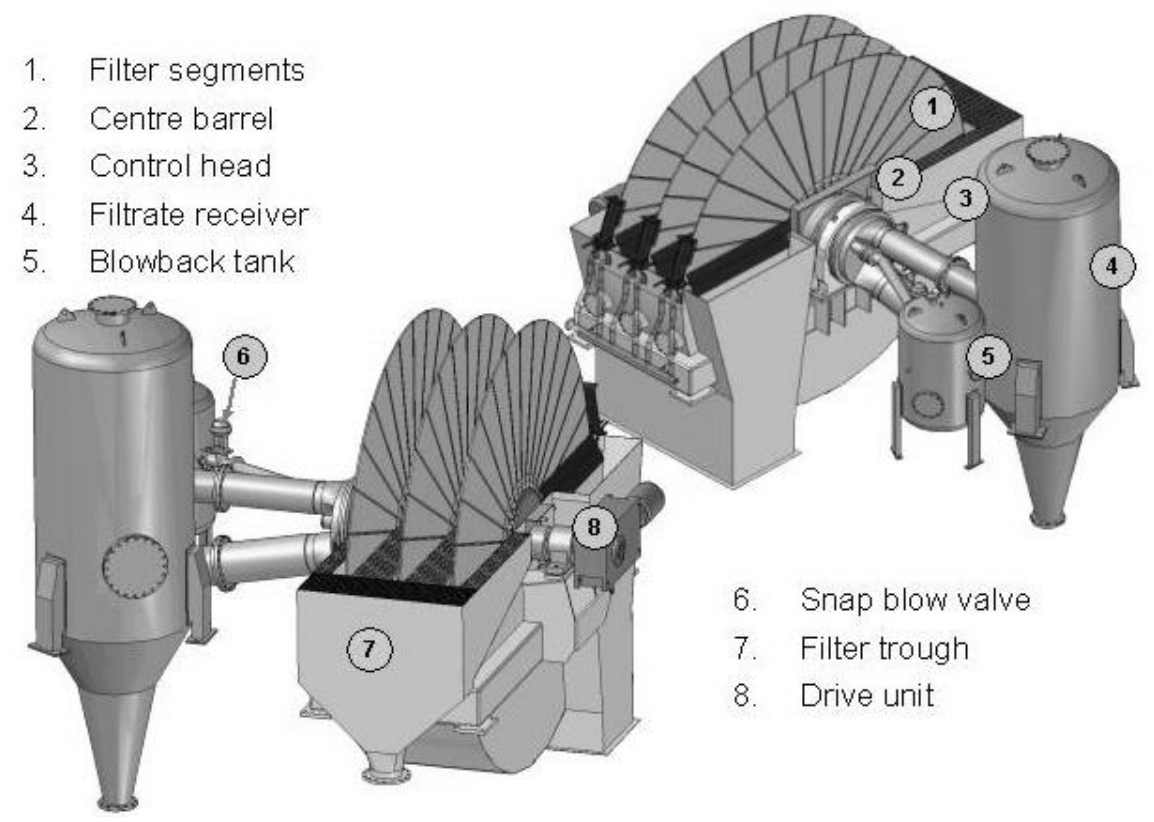

Figure 1 View of a Boozer disc filter with three discs 


\subsection{Main components}

The Boozer is designed by optimising each detail consequently according to flow requirements. This way pressure loss in the whole flow route from the filter segments up to the filtrate receivers has been minimised.

\subsubsection{Robust filter segments}

Filter discs consist of 30 segments with robust construction and a low weight of less than $20 \mathrm{~kg}$ per segment. By having 30 segments per disc leads to small sized segments ensuring optimal hydraulic conditions for cake formation, fast filtrate drainage and complete cake discharge in less than 0.2 seconds without filtrate blowing back, i.e. without cake re-wetting. On the other side, this number of low weight segments is ideal to facilitate re-clothing and maintenance. The segment bell is made for a fast, easy and secure filter bag fixing and is designed in a shape that allows fixing of the filter bags with cable ties. This fast and secure method simplifies filter cloth fixing and reduces the time needed for re-clothing.

\subsubsection{Pre-separation control head}

The two-phase-flow of filtrate and air, which enters the pipe system in the cake dewatering zone, together with the high flow velocity are the important factors for the pressure loss. Therefore, the control head of the Boozer (Figure 2) is designed to pre-separate filtrate and air already inside the control head. The outlet nozzles are a specially streamlined shape to minimise flow restrictions and pressure loss. The control head is pressed against the wear plate via a central tightening. It is not supported on the trough construction (as is standard filter design) but on the shaft centre which leads to a short closed linkage.

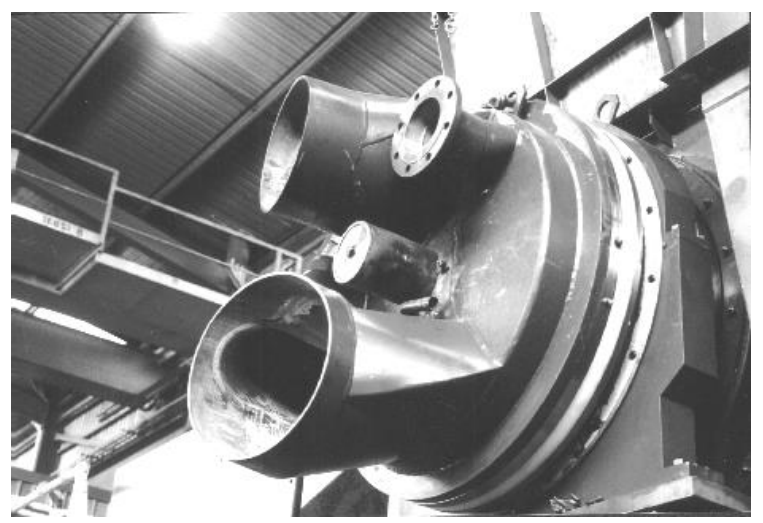

Figure 2 Pre-separation control head of the Boozer

\subsubsection{Filter arrangement}

Between the control head and filtrate receiver the mentioned two-phase-flow of filtrate and air can lead to a high pressure loss, $\Delta \mathrm{p}$, of up to ten times higher than a one-phase-flow. Hence, the arrangement of the filter and the filtrate receivers' influences filter operation and filter performance. Therefore, the Boozer disc filter and the filtrate receiver are located as close and straight as possible to keep this flow section very short (see Figure 1). The layout of the pipes between control head and receiver avoids any bend as far as possible and the receiver inlet is carried out tangentially.

\subsection{4 “Joint single" trough without agitator}

The "joint single" trough (Figure 3) of the Boozer has been designed to combine the advantages of the common trough design (all discs run in a common trough) and the single trough design (every filter disc runs in its own single trough) without the respective disadvantages and operation failures as described by Bott et al. (2004). The joint single trough is constructed like a common trough with installations between the discs at the slurry inlet side which subdivide the trough in narrow compartments for each disc. Thus, it works without an agitator (like the standard single trough) since the stirring effect of the discs rotating in narrow compartments homogenises the slurry. Like with the common trough design all discs run with the same slurry level thanks to a large sized, common overflow. The free exchange of slurry between the single trough 
compartments ensures a common level in the trough for all discs. The design and arrangement of the feed manifold is such the incoming slurry supports the stirring effect of the rotating discs.

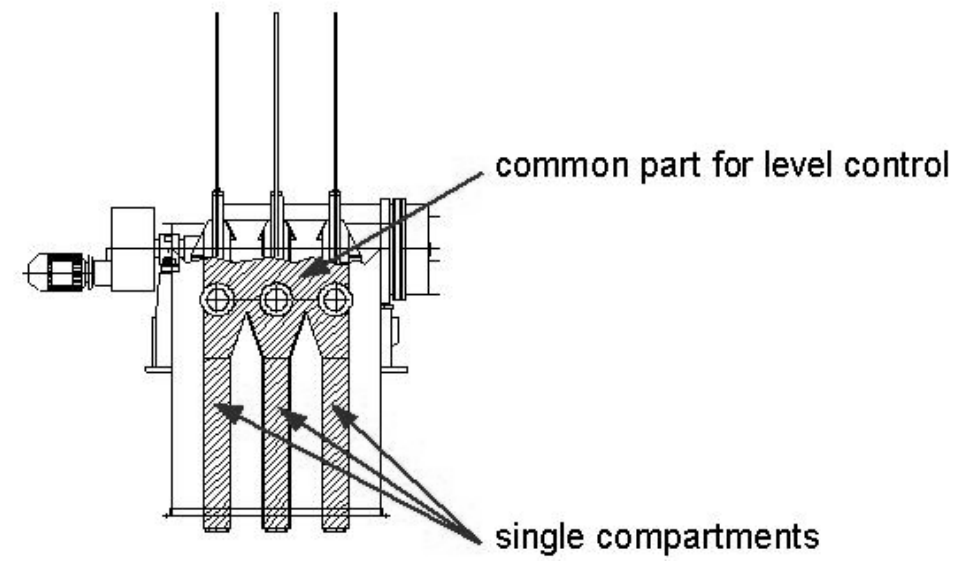

Figure 3 Joint single trough - all discs run with the same slurry level

\subsubsection{Cake discharge and cake deflector boxes}

The filter cake is discharged by a compressed air blowback which is precisely timed by a snap blow valve. The small sector volume and the hydraulic design provide for quick filtrate drainage and for a quick and intensive blowback impulse with only $0.25-0.35$ bar overpressure and without blowing back of filtrate into the cake. Even at high filter speed when thin filter cakes of only $4-5 \mathrm{~mm}$ in height are produced the cake is totally discharged. The Boozer can therefore be satisfactorily operated with thin filter cakes at a high filter speed of up to $6 \mathrm{rpm}$.
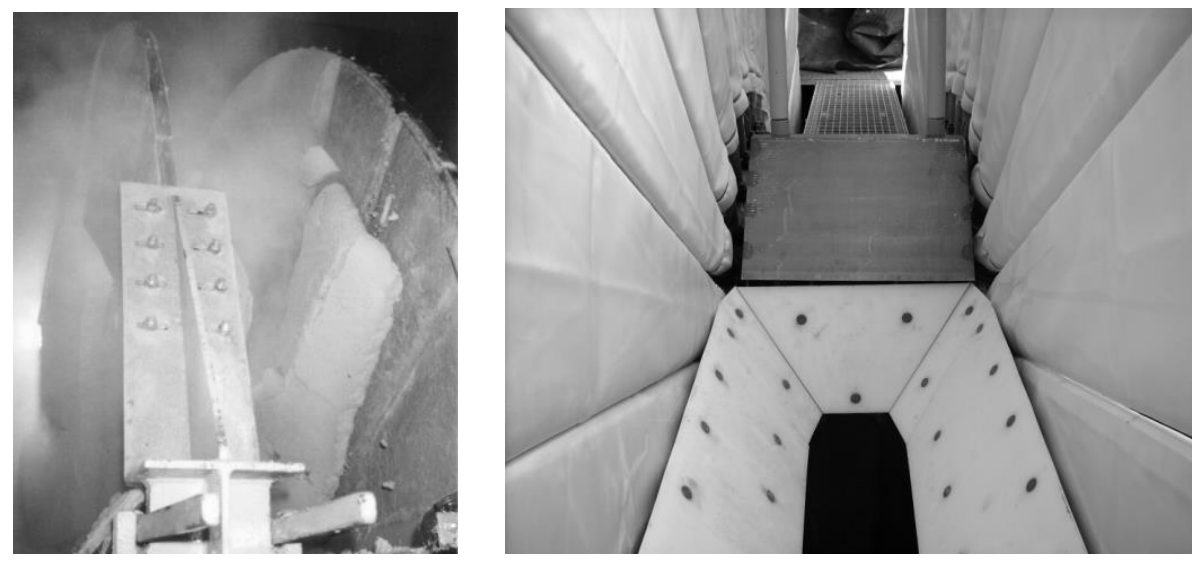

Figure 4 Complete cake discharge (left), advanced scraper design (right)

Cake deflector boxes with anti-friction tops made of ultrahigh modulus polyethylene (UHMPE) (Figure 4) prevent accumulation of cake and ensure an exact adjustment of the distance between cake deflector and filter disc (in conjunction with disc guide rollers), i.e. no filter cake falls back into the trough and filter performance keeps constantly high.

\subsection{Operation and maintenance}

\subsubsection{Vacuum system}

For the layout of the vacuum system two alternatives are possible. Each filter can be connected to an own, separate vacuum pump (single vacuum system) or all filters are connected to a common vacuum line (common vacuum system) as schematically shown in Figure 5. 


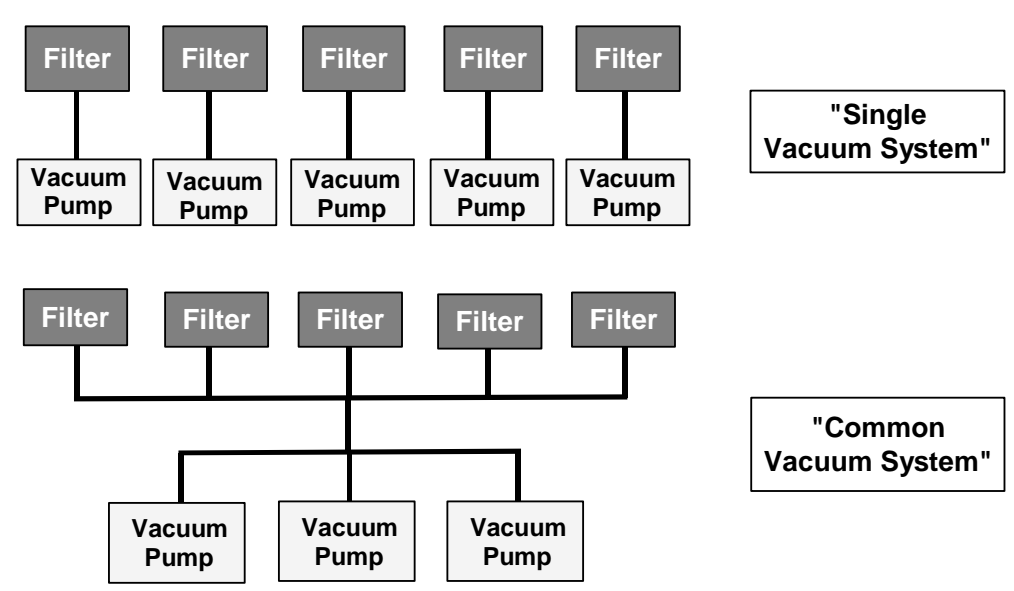

Figure 5 Single and common vacuum system for a seed filter station

Due to the reliable operation control Boozer filters can be operated with the common vacuum system. This system provides for higher availability and operation security of the filter plant compared to the single vacuum system because failure of a pump is compensated by the other parallel working pumps and does not affect the overall filter performance. Furthermore, the installation of a few larger sized pump units is cheaper than the installation of a multitude of smaller sized pumps with respect to invest and maintenance cost. However, this superior vacuum system can only be used in combination with a filter control system as developed and implemented by Bokela which prevents that failure of one filter (e.g. if a filter sucks air due to sinking slurry level in the trough) affects the whole vacuum line.

\subsubsection{Process and control philosophy}

The design and the outstanding hydraulic capacity of the Boozer is the basis of the Bokela Operation Philosophy for improved filter operation without continuous slurry overflow.

An automatic filter operation control adapts filter performance to changing slurry and process conditions and avoids emergencies. A primary and a secondary control circuit ensure a self-regulating and secure filter operation by using the slurry level in the trough as the control variable and the filter speed and the pressure difference as the control output. The possibility to run the Boozer with a high filter speed of $n=6 \mathrm{rpm}$ and the high pressure difference, which is available at the filter cloth, allow filter operation in a wide performance range. All important functions and peripheral components like vacuum pump, filter drive, trough level, blower, the vacuum in the receivers, pressure in the cake blow off tank, lubrication pump, etc., are supervised and integrated in the interlock schedule. In case of an emergency the PLC gives an alarm and an adequate procedure is started that depends on the respective emergency case. In any case the drain valve of the trough opens, the filter is put off from the vacuum and the feed valve is closed in order to avoid that the slurry floods the filter floor.

\subsubsection{Primary control circuit - trough level control by adjusting of the filter speed}

The slurry level is measured continuously by a proven radar measurement device which is suitable even for foam on the slurry surface and mist/vapour in the air. The signal of the level measurement is used to adjust the variable filter speed by a variable frequency controller (Figure 6).

At normal operation the filter is fed with a more or less constant slurry flow and the Boozer operates with a medium filter speed of approximately 2.0-3.0 rpm and a fully open vacuum control valve in the vacuum line (form zone) between control head and filtrate receiver. The best performance of the filter is achieved if the slurry level in the filter trough is close to the overflow level (approximately 50\% submergence of the filter disc). In this case the cake formation angle is maximum and an even filter cake with a constant cake thickness over each segment is formed. This results in very constant operation conditions. 


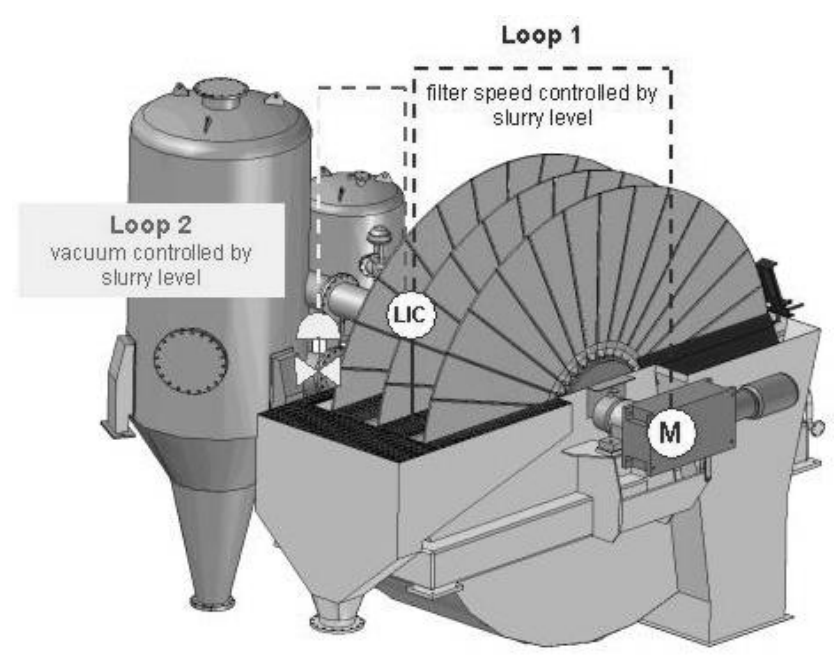

Figure 6 Schematic filter operation control system of the high performance disc filter Boozer with fully automatic operation

If the slurry level in the filter trough drops or increases due to a changing slurry flow or changing product characteristics, the filter speed is automatically reduced or increased in order to maintain a constant high slurry level in the trough (level set point). If the filter runs at minimum permitted speed and the slurry level in the trough still drops, then the second control circuit becomes active and the vacuum in the filter cake formation zone is adapted accordingly.

\subsubsection{Secondary control circuit - trough level control by adjusting of the form zone vacuum}

The control of the vacuum in the cake formation zone with the vacuum control valve is the second control circuit (Figure 6). The target is to maintain a constant and high slurry level in the trough even at minimum filter speed. This control circuit becomes active if the filter runs with minimum speed and the slurry level in the trough still drops. Then, the vacuum control valve in the vacuum pipe (form zone) between filter and filtrate receiver is closed as needed to maintain the level set point in the trough. If the form zone vacuum control valve is closed to the limit and the filter speed is already at the minimum but the slurry level still drops, then, the trough level can be reduced to a minimum permitted set point to further reduce the filter performance.

The individual vacuum control in the form zone also enables to maintain the required cake moisture in case of changing filtration properties of the tailings by reducing the vacuum in the form zone. This way a filter setting i.e. a ratio of cake drying angle $\alpha_{2}$ to cake formation angle $\alpha_{1}$ of $\alpha_{2} / \alpha_{1}=1-5$ can be attained.

\subsubsection{Double dewatering zone}

For tailing filtration the Boozer is equipped with a double dewatering zone which can be activated or deactivated online to allow reacting online on changes in the filtration properties of the thickened tailings. In case the filter cake is building cracks which then leads to a high air consumption or even a vacuum breakdown the second dewatering zone can be deactivated maintaining stable filter operation with somewhat higher moisture content in the filter cake.

\subsubsection{Maintenance}

Boozer disc filters are of robust design, they do not require a lot of maintenance and ensure a reliable operation with high filter availability. Maintenance work is simplified, manpower and costs for maintenance are widely reduced compared to disc filters of standard design due to a lot of special design features and improved details such as:

- walkways between the filter discs (see Figure 7) make maintenance work more comfortable

- segments with low weight that are easy to exchange thanks to bayonet fixing 
- re-clothing (a main part of maintenance work) is simplified while the cloth lifetime is prolonged

- cloth wash system with removable wash bars

- no agitator thanks to joint single trough design, i.e. no exchange of agitator sealings

- maintenance free barrel sealing needs no exchange but only flushing during filter cleaning

- long lifetime of wear plate of 4-5 years

- anti-friction tops made of UHMPE on cake deflector boxes (carbon steel) with long lifetime.

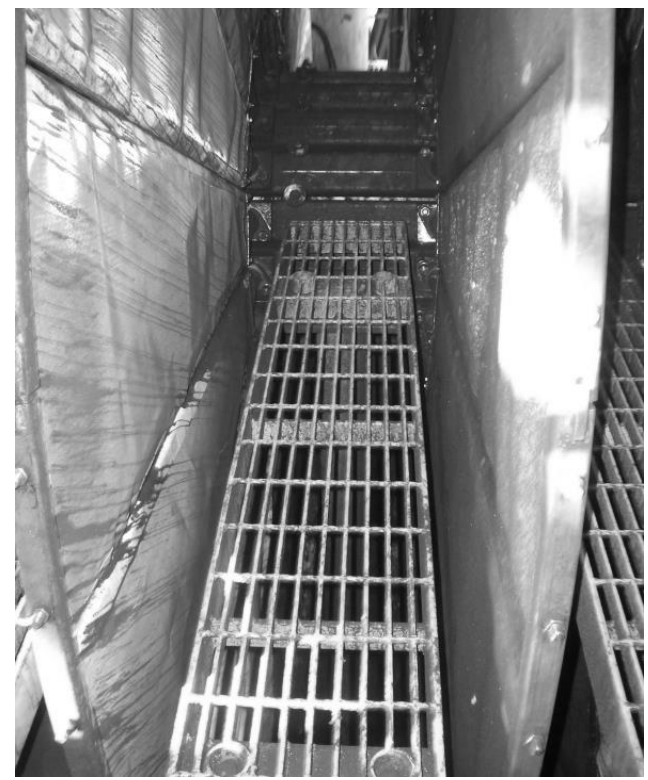

Figure 7 Walkways between the filter discs permit easy and safe access to segments for quick reclothing

\section{Filtration of the gold/copper tailings with the Boozer disc filter}

\subsection{Filtration tests for filter layout}

In the particular case of a copper and gold mine the tailings should be used for mine backfill. The tailings consist of particles with a mean diameter size of $\mathrm{d}_{50}$ of 20-30 micron. They are thickened to $>50 \mathrm{wt} \%$ in a thickener. Filtration tests with two tailing samples (one with a $\mathrm{d}_{50}$ close to 20 micron and one with a $\mathrm{d}_{50}$ close to 30 micron) have been carried out. First of all the amount of flocculant required to achieve a reasonable solids throughput was investigated. Figure 8 shows the cake formation time plotted versus the flocculant dosage. With increasing flocculant dosage the cake formation time decreases which will result on higher solids throughput rates on the filter. The graph shows that above $100 \mathrm{~g} / \mathrm{t}$ flocculant there is no further significant improvement on the cake forming time. Therefore, a flocculant dosage of $75 \mathrm{~g} / \mathrm{t}$ was chosen for the rest of the tests.

Next the achievable residual moisture of the filter cake was investigated. The target moisture for the process is $23 \mathrm{wt} \%$. Figure 9 shows the moisture content plotted versus the dewatering ratio which is the adjusted time (or angle $\alpha_{2}$ ) on the rotary disc filter for cake drying divided by the adjusted time (or angle $\alpha_{1}$ ) of the filter for cake formation. Typically, for rotary disc filters the ratio $\alpha_{2} / \alpha_{1}$ ranges from 1 to 2 , i.e. the time available for cake dewatering can be the double of the time available for cake formation. With the coarse Sample A the moisture target is already reached at a dewatering ratio of 0.65 while the Sample B requires a dewatering ratio of 1.0 to hit the target. This allows for a filter setting with cake formation time/angle to be the same as cake dry time/angle $\left(\alpha_{2} / \alpha_{1}=1\right)$. For this setting further filtration tests have been carried out to investigate the resulting specific solids throughput with this setting. The results are shown in Figure 10. Filter cake height for this setting ranges from 8-15 mm depending on filter speed. With the individual vacuum control in the form zone the Boozer disc filter enables to vary the filter setting online even to a ratio of cake drying angle $\alpha_{2}$ to cake formation angle $\alpha_{1}$ of $\alpha_{2} / \alpha_{1}=1-5$. 
The test results show that the finer Sample A is reaching $650 \mathrm{~kg} / \mathrm{m}^{2} / \mathrm{h}$ specific solids throughput and the coarser Sample B is reaching $800 \mathrm{~kg} / \mathrm{m}^{2} / \mathrm{h}$ specific solids throughput. Therefore, the required $100 \mathrm{t} / \mathrm{h}$ solids throughput can be achieved with one Boozer L4 disc filter with $176 \mathrm{~m}^{2}$ filter area which requires a minimum specific solids throughput of $568 \mathrm{~kg} / \mathrm{m}^{2} / \mathrm{h}$. The chosen filter size in fact allows for a peak throughput of 114-141 t/h which leaves a significant safety margin in the filter sizing. Depending on the modern design and operational flexibility this extra capacity can be converted into improved moisture during filter operation (on line).

Cake Formation Time Depending on Flocculant Dosage

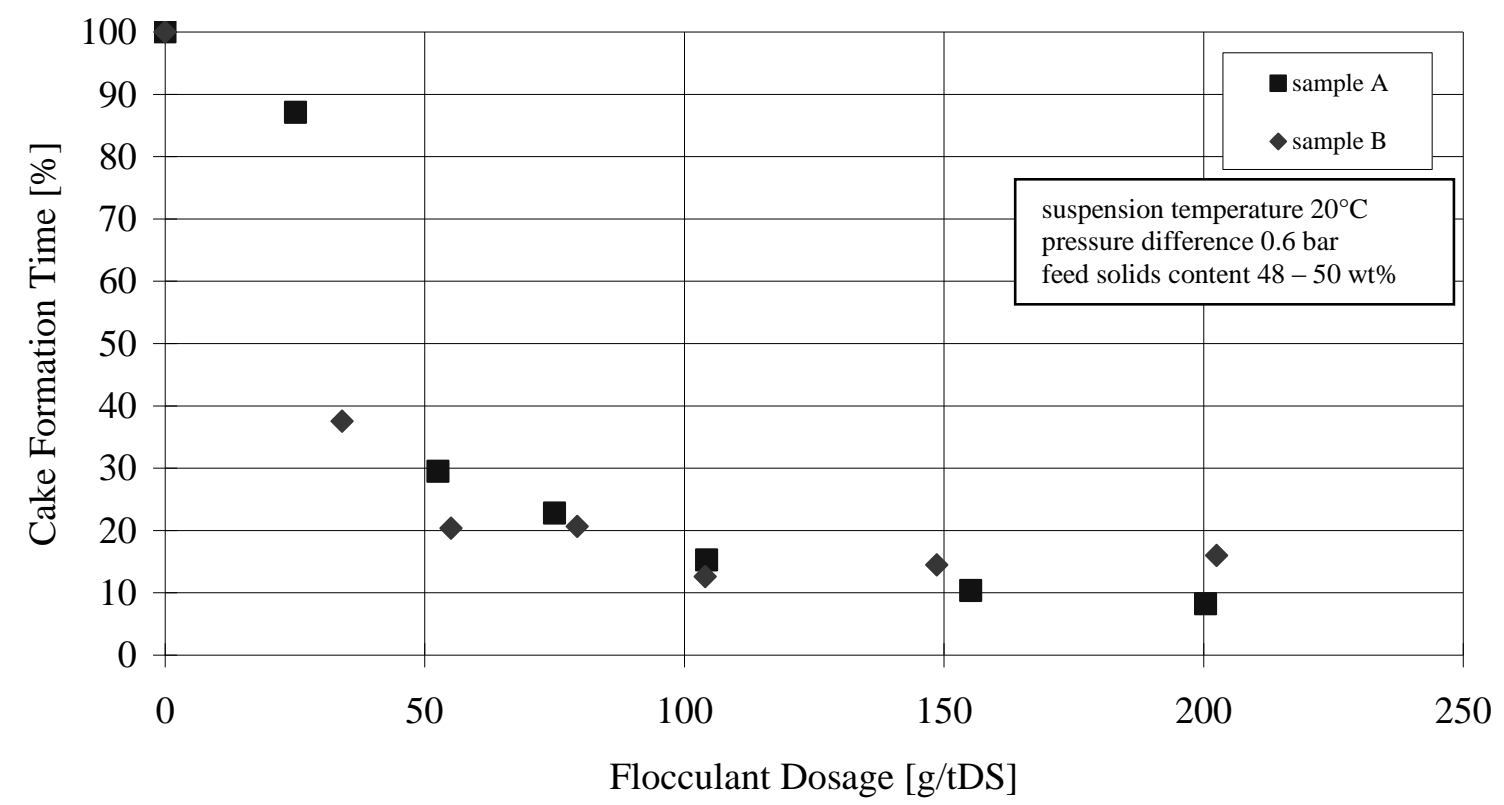

Figure 8 Moisture content plotted versus flocculant dosage (a flocculant dosage of $75 \mathrm{~g} / \mathrm{t}$ was chosen for the rest of the tests)

Moisture Content Depending on the Dewatering Ratio

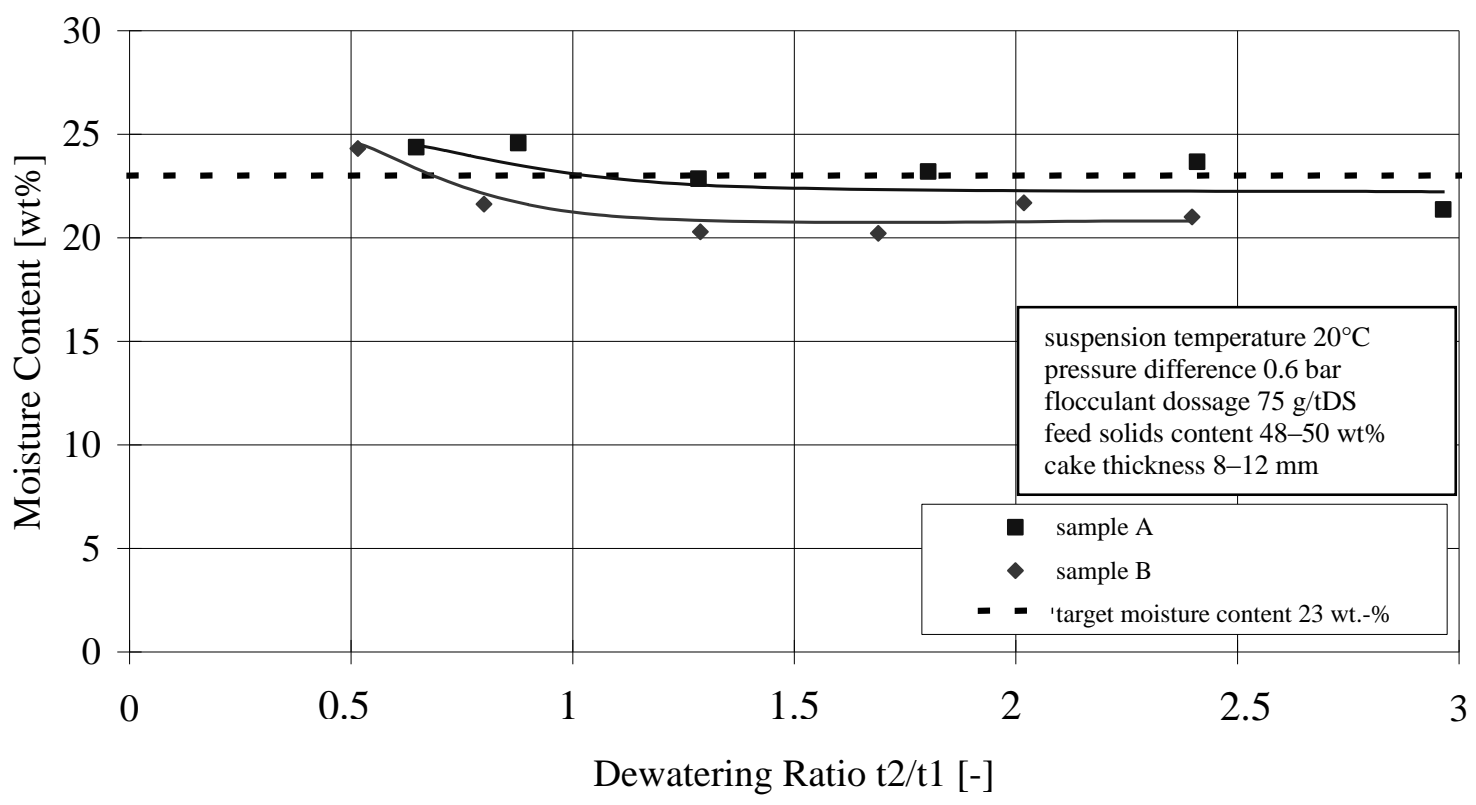

Figure 9 Moisture content as function of dewatering ratio which is the ratio of cake formation angle $\alpha_{1}$ to cake dry angle $\alpha_{2}$ 
Specific Solid Throughput Depending on Filter Speed

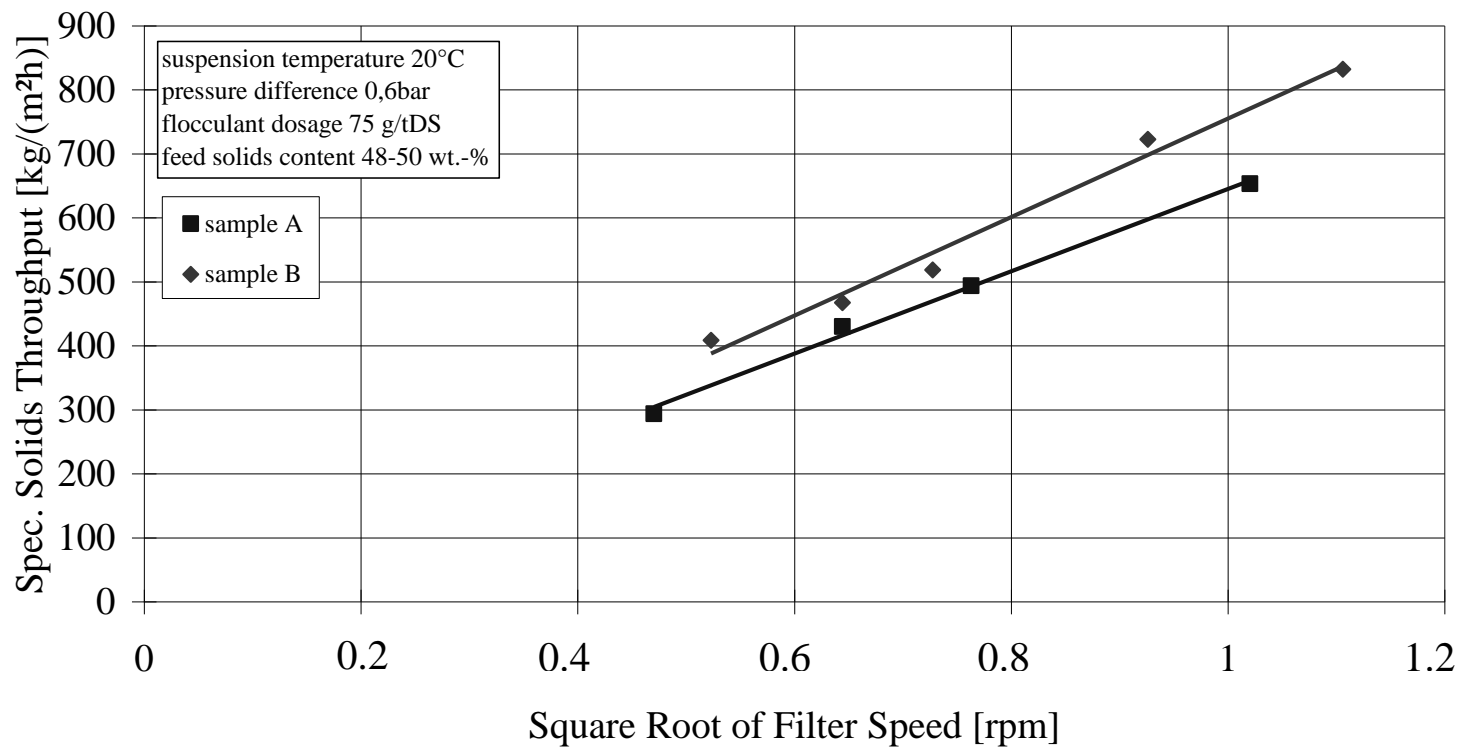

Figure 10 Specific solids throughput versus square root of filter speed (with chosen filter setting of cake dry angle to cake formation angle $\alpha_{2} / \alpha_{1}=1$ the height of filter cakes ranges from 8-15 mm)

\subsection{Experience of filter commissioning}

According to the filtration tests results a Boozer L4 disc filter with $176 \mathrm{~m}^{2}$ filter area has been chosen as appropriate filter size for this tailing filtration task (Figure 11). During commissioning of the filter the thickener was not in constant operation. Therefore, the filter was fed with feed solids down to $25 \mathrm{wt} \%$ and almost no flocculant added. Due to these harsh conditions the filter was building only a cake of 3-5 mm thickness. But nevertheless, the filter was discharging almost all of the solids and the moisture content was almost below $20 \mathrm{wt} \%$.
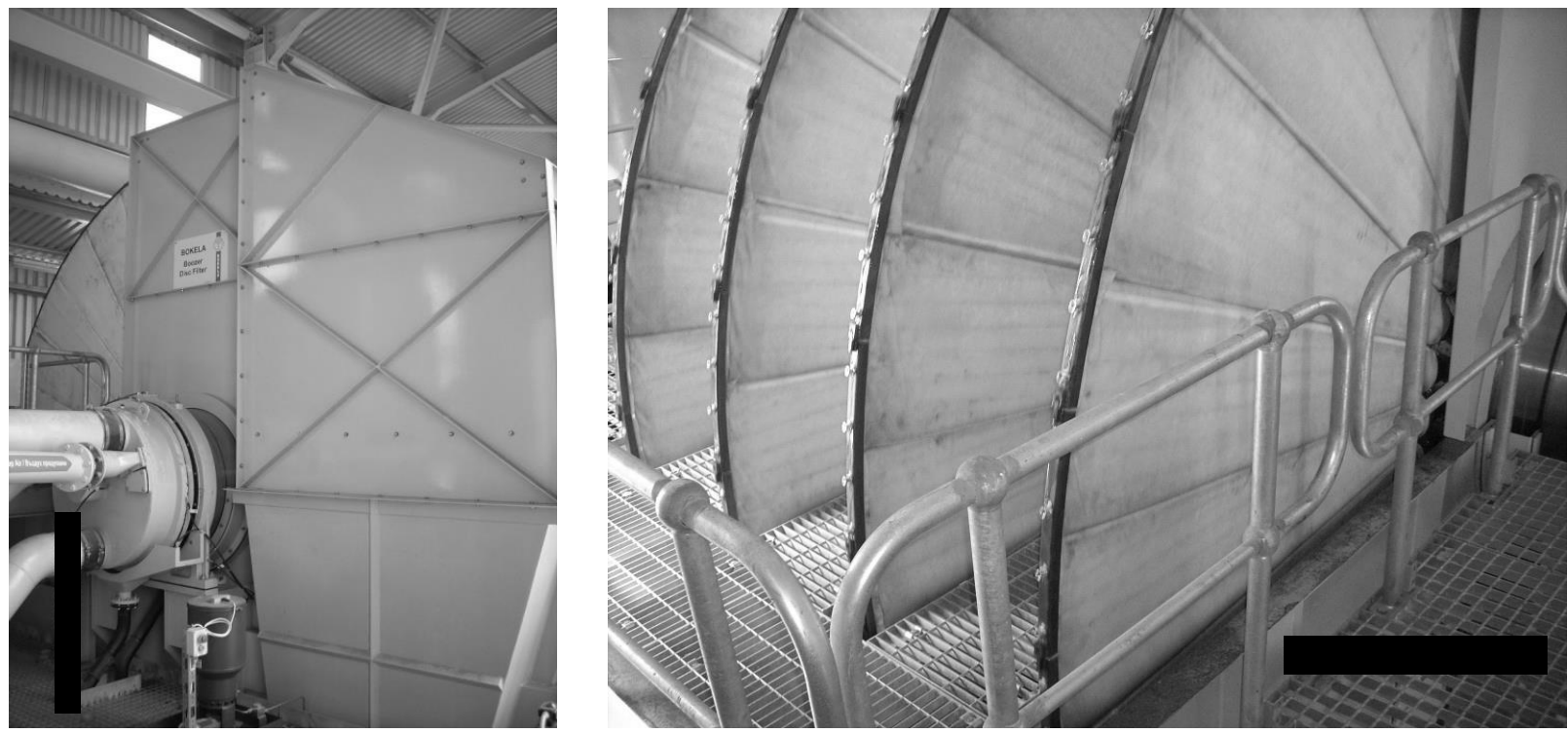

Figure 11 Boozer L4 disc filter with $176 \mathrm{~m}^{2}$ filter area assembled in the gold/copper mine. View of control head side (left) and view of the four filter discs with walkways (right) 
After flocculant was added the cake thickness increased immediately to more than $10 \mathrm{~mm}$ (sometimes more than $20 \mathrm{~mm}$ ) and the specific solids throughput was up to almost $1,000 \mathrm{~kg} / \mathrm{m}^{2} / \mathrm{h}$ with a moisture in the range of $21-23 \mathrm{wt} \%$.

\section{$5 \quad$ Filtration results with other tailings}

Similar filtration results have been achieved in other copper/gold projects as well as with tailings from other base areas like lead and zinc or even with coal tailings from South Africa as long as there is no significant clay content. Figure 12 shows the moisture content of filtered tailings for different copper/gold tailings. The results show very well that the moisture decreases with increasing particle size of the feed material. Therefore, the PSD of the tailings already gives a good indication of the moisture that can be expected on the filters.

The specific solids throughput of various tailings is also very similar as Figure 13 demonstrates. Solids throughput of $500 \mathrm{~kg} / \mathrm{m}^{2} / \mathrm{h}$ seems to be a conservative figure which may fit for almost all these tailings while some allow for up to $1.000 \mathrm{~kg} / \mathrm{m}^{2} / \mathrm{h}$. The reason why there is very little difference in the specific solids throughput is mostly depended on the amount of flocculant added which is usually dosed in an amount suitable to get into this $500-1,000 \mathrm{~kg} / \mathrm{m}^{2} / \mathrm{h}$ range. Subsequently, this flocculant dosage varies from $10-100 \mathrm{~g} / \mathrm{t}$.

But again, if the tailings contain significant amounts of clay, then the specific solids throughput reduces to a tenth or less of the above figures and the moisture increases to the high $20 \mathrm{~s}$.

Moisture Content Depending on the Dewatering Ratio

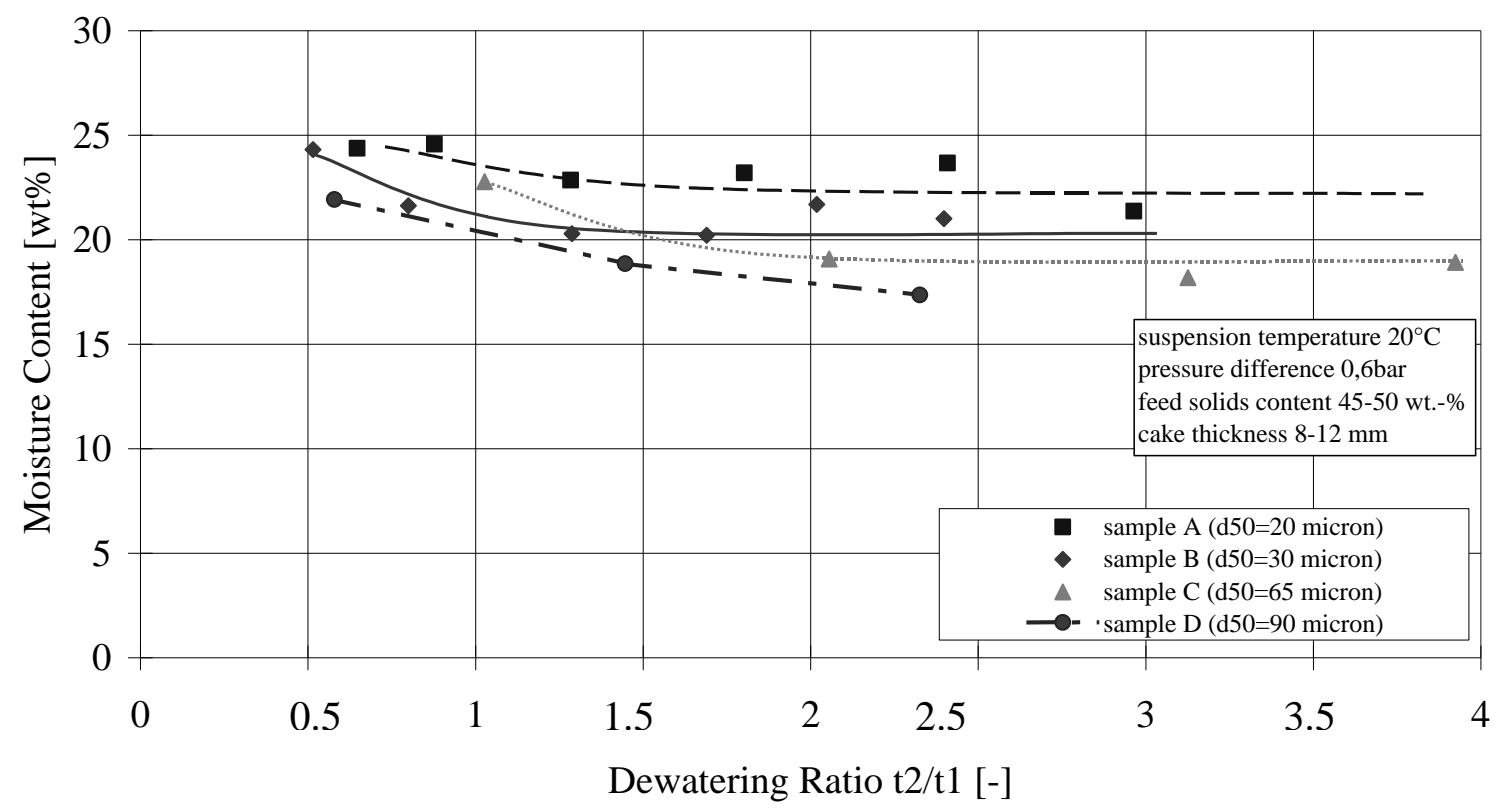

Figure 12 Moisture content of filtered tailings for different copper/gold tailings 
Specific Solid Throughput Depending on Filter Speed

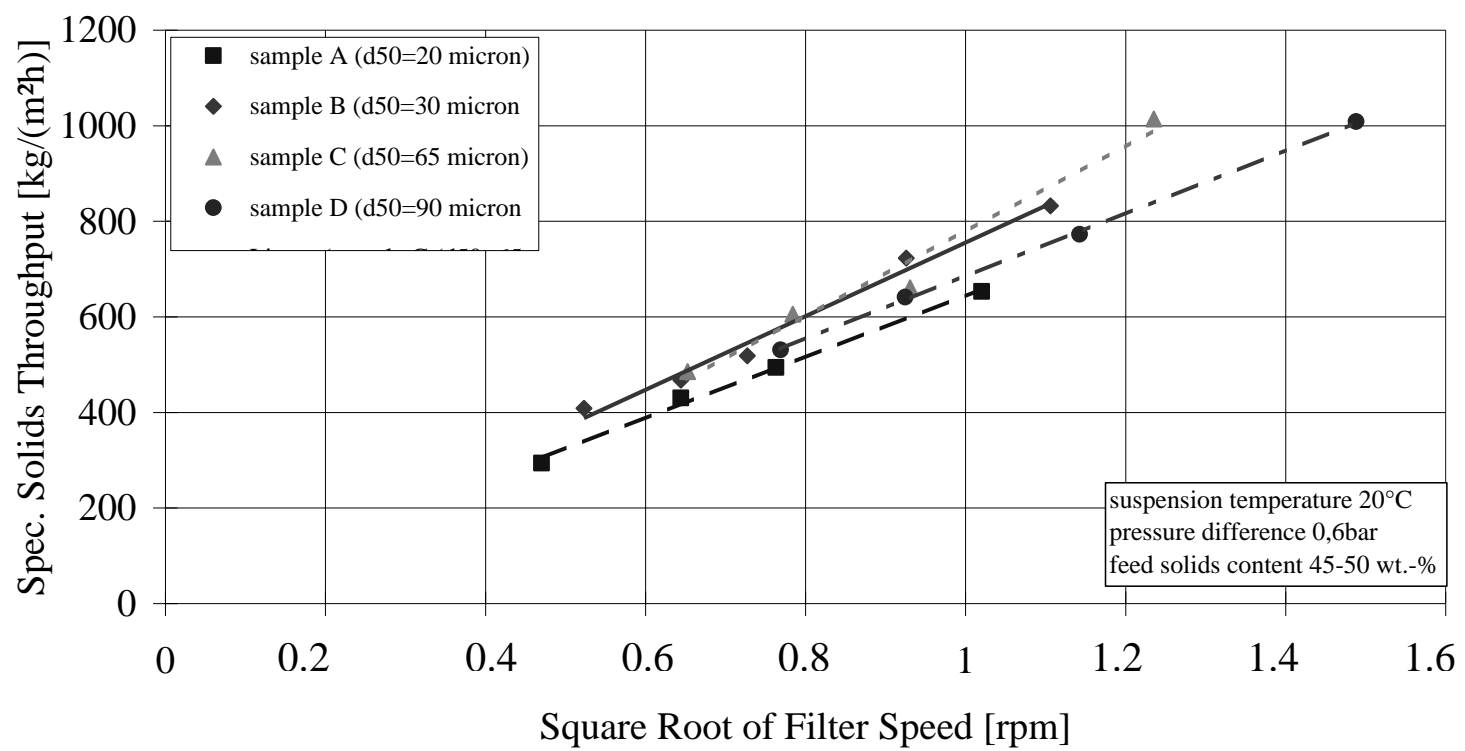

Figure 13 Specific solids throughput of filtered tailings for different copper/gold tailings

\section{Acknowledgements}

The authors thank the clients for trusting in Bokela's solid/liquid separation technologies and engineering services.

\section{References}

Bott, R., Langeloh, T. and Hahn, J. (2004) Latest state of the art in Al-hydrate filtration, in Proceedings 15th International Symposium of ICSOBA - Aluminium Industry within World Economy: Problems and Trends of Development, St. Petersburg, Russia, June 15-18 2004, pp. 177-184. 
